\title{
Anomalous Momentum Dependence of the Quasiparticle Scattering Rate in Overdoped $\mathrm{Bi}_{2} \mathrm{Sr}_{2} \mathrm{CaCu}_{2} \mathrm{O}_{8}$
}

\author{
P.V. Bogdanov, ${ }^{1}$ A. Lanzara, ${ }^{1,2}$ X. J. Zhou, ${ }^{1}$ W. L. Yang, ${ }^{2}$ H. Eisaki, ${ }^{1}$ Z. Hussain, ${ }^{2}$ and Z. X. Shen ${ }^{1}$ \\ ${ }^{1}$ Department of Physics, Applied Physics and Stanford Synchrotron Radiation Laboratory, Stanford University, \\ Stanford, California 94305 \\ ${ }^{2}$ Advanced Light Source, Lawrence Berkeley National Laboratory, Berkeley, California 94720
}

(Received 7 February 2002; published 26 September 2002)

\begin{abstract}
The question of the anisotropy of the electron scattering in high temperature superconductors is investigated using high resolution angle-resolved photoemission data from $\mathrm{Pb}$-doped $\mathrm{Bi}_{2} \mathrm{Sr}_{2} \mathrm{CaCu}_{2} \mathrm{O}_{8}$ (Bi2212) with suppressed superstructure. The scattering rate of low energy electrons along two bilayersplit pieces of the Fermi surface is measured (via the quasiparticle peak width), and no increase of scattering towards the antinode $(\pi, 0)$ region is observed, contradicting the expectation from $Q=$ $(\pi, \pi)$ scattering. The results put a limit on the effects of $Q=(\pi, \pi)$ scattering on the electronic structure of this overdoped superconductor with still very high $T_{c}$.
\end{abstract}

DOI: $10.1103 /$ PhysRevLett.89.167002

PACS numbers: 74.72.-h, 74.25.-q

The momentum dependence of the "quasiparticle" width along the Fermi surface is a very sensitive test of the scattering processes in cuprates, which is ultimately important to understand its superconducting mechanism. In the underdoped regime in $p$-type cuprates [1-4], it has been shown in many systems that the spectra near $(\pi, 0)$ is particularly broad. This anisotropy is generally attributed to spin scattering centered at $Q=(\pi, \pi)$, that for $p$-type cuprates in the $k$ space connects $(\pi, 0)$ to $(0, \pi)$ points of the Brillouin zone - the Fermi surface "hot spots." In optimal and overdoped $n$-type materials, the $Q=(\pi, \pi)$ vector also connects Fermi surface points, and corresponding hot spots were recently reported in $\mathrm{Nd}_{1.85} \mathrm{Ce}_{0.15} \mathrm{CuO}_{4}$ (NCCO) [5]. The $Q=(\pi, \pi)$ scattering and its broadening of the $(\pi, 0)$ spectra exhibit itself most strongly in the undoped insulator $[6,7]$. There is a general agreement that insulating behavior in the undoped system results from a strong electron-electron correlation effect which is also intimately related to antiferromagnetic interactions peaked at $Q=(\pi, \pi)$. Hence, an anisotropy in peak width which increases towards $(\pi, 0)$ in $p$-type materials is a hallmark of the impact on the electron dynamics by $Q=(\pi, \pi)$ antiferromagnetic interaction. Since this effect is generally expected on theoretical grounds and is seen in many materials, it remains true despite two technical problems complicating the analysis of the $\mathrm{Bi} 2212$ data near $(\pi, 0)$. One is the superstructure, and the other is the recently realized bilayer splitting [8$11]$ that strongly influences the near $(\pi, 0)$ spectra when it is unresolved.

The scattering anisotropy in overdoped $p$-type samples has hardly been studied, other than a brief discussion of Bi2212 in [1], and the resolution was not good enough for quantitative analysis. In this Letter, we present the first results of scattering rate measurements along the two well-resolved pieces of the Fermi surface in overdoped $\mathrm{Pb}$-doped Bi2212 with suppressed superstructure. As shown in Fig. 1, these samples and experimental setup allow clear observation of the two bilayer-split Fermi surfaces using angle-resolved photoemission spectroscopy (ARPES) [10]. We performed measurements above and well below the superconducting transition temperature to measure the widths of the quasiparticle peaks along the two resolved Fermi surface pieces and found that the scattering rate does not increase towards $(\pi, 0)$. This contradicts what is expected from $Q=(\pi, \pi)$ scattering, indicating that the hot spot has already turned cold at this doping level. This strongly suggests that any magnetic excitations near $Q=(\pi, \pi)$ would have minimal effect on the quasiparticle dynamics, consistent with $[12,13]$, but inconsistent with [14-17]. This finding in a sample with $T_{c}$ as high as $70 \mathrm{~K}$ puts a very strong constraint on theory. In particular, it shows that the $Q=$ $(\pi, \pi)$ magnetic resonance mode cannot be the driving force for pairing.
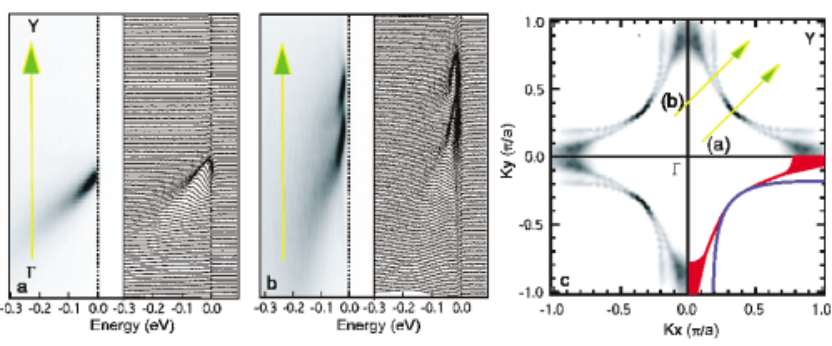

FIG. 1 (color). Panel (a) of this figure shows raw ARPES data along the $\Gamma-Y$ scan. In panel (b), we plot data for the parallel cut $10^{\circ}$ off the $\Gamma-Y$ direction. In panel (c), an eightfold symmetrized map of the spectral intensity at $12 \mathrm{meV}$ binding energy in superconducting Bi2212 is presented. This map is representative of the Fermi surface situation in Bi2212. The lower right quadrant of the Brillouin zone in panel (c) identifies the bonding band Fermi surface (blue) and the antibonding band Fermi surface (red). 
ARPES data have been recorded at beam line 10.0.1 of the Advanced Light Source utilizing $22 \mathrm{eV}$ photon energy in $4 \times 10^{-11}$ Torr or better vacuum. The sample was kept in the fixed position relative to the beam polarization, and the analyzer was rotated. The beam polarization was in the sample plane perpendicular to the $\Gamma-Y$ direction, with the beam nearly at grazing incidence with the sample surface. We used a Scienta SES 200 analyzer in the angle mode, where cuts parallel to the $\Gamma-Y$ direction were carried out. Momentum resolutions were $\pm 0.004 \AA^{-1}$ along the cut and $\pm 0.011 \AA^{-1}$ perpendicular to the cut, and the energy resolution was $14 \mathrm{meV}$. The overdoped $\mathrm{Pb}$ doped Bi2212 $\left(T_{c}=70 \mathrm{~K}\right)$ was grown using the floatingzone method. The Fermi energy was obtained from the energy distribution curves (EDCs) of polycrystalline Au.

Panel (a) of Fig. 2 shows the photoemission intensity map taken at the Fermi level at $20 \mathrm{~K}$, well below the superconducting transition temperature of $70 \mathrm{~K}$. One can clearly see two Fermi surface pieces, which coincide along the diagonal direction $[(0,0)-(1,1)$ in the units of $(\pi / a)]$, and separate significantly at $(1,0)$, as expected from the interaction between two adjacent $\mathrm{CuO}_{2}$ planes [18]. The clearly resolved bilayer splitting and the suppressed superstructure effect near the $(\pi, 0)$ region (due to $\mathrm{Pb}$ doping) make the following analysis possible.

Crosses on the map in panel (a) indicate momentumspace points where spectra shown in panels (b)-(e) were taken. Blue spectra in panels (b)-(e) are taken along the bonding Fermi surface at the $b 1-e 1$ points of panel (a). One sees that on going along the bonding Fermi surface spectra does not change significantly. The width of the peak appears almost constant independent of the position in momentum space, strongly hinting that the quasipar-

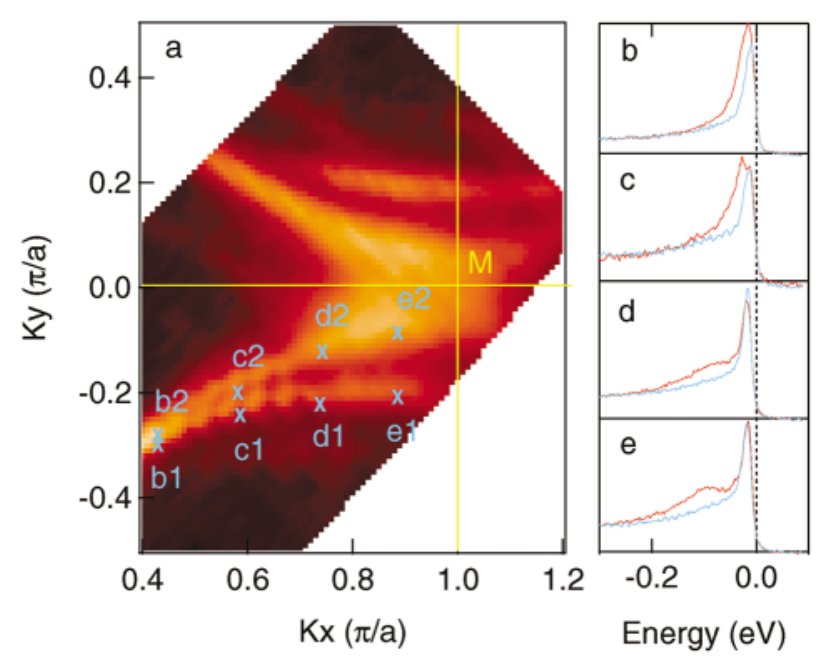

FIG. 2 (color). In this figure data collected at $20 \mathrm{~K}$ is shown. Panel (a) shows the spectral intensity at the Fermi level. Crosses indicate locations in momentum space of the cuts shown in panels (b)-(e). Blue spectra come from the bonding band, while red spectra come from the antibonding band. ticle scattering rate is more or less isotropic [19]. Red spectra in panels (b)-(e) are taken along the antibonding Fermi surface at $b 2-e 2$ points in panel (a). Here the structure is more complex, but it is clear that it can be reproduced by two features: a peak at the Fermi level and a feature that disperses to higher binding energy as one moves from the $(0,0)-(1,-1)$ direction toward the $(0,0)-(1,0)$ direction. The former comes from the antibonding band at the Fermi surface, and the latter results from the bonding band [8-10]. It is interesting to note that the low energy antibonding band peak is very similar to the bonding band peak at the Fermi surface. By plotting spectra from both bands in one graph, we see that the low energy signal is similar for both Fermi surfaces.

Figure 3 plots the data at $100 \mathrm{~K}$, well above the superconducting transition temperature of $70 \mathrm{~K}$. Again, panel (a) shows the intensity map at the Fermi level, while panels (b)-(e) show individual spectra along the two Fermi surfaces. Similar to the results of Fig. 2, the bonding band gives rise to one-peak structure at the Fermi level. The antibonding Fermi surface results can be represented as the sum of the low energy peak, similar to the peak from the bonding band, and the dispersing higher energy feature, as illustrated in panels (b)-(e).

To address the above discussion in more detail, we use several ways to compare in detail the spectral widths along the Fermi surfaces. We use the EDC rather than momentum distribution curve (MDC) widths to determine the scattering rate. It is reasonable in this case, because the peaks are relatively sharp and we are mainly interested in relative change with momenta, not necessarily the absolute value. Further, the flat band near $(\pi, 0)$

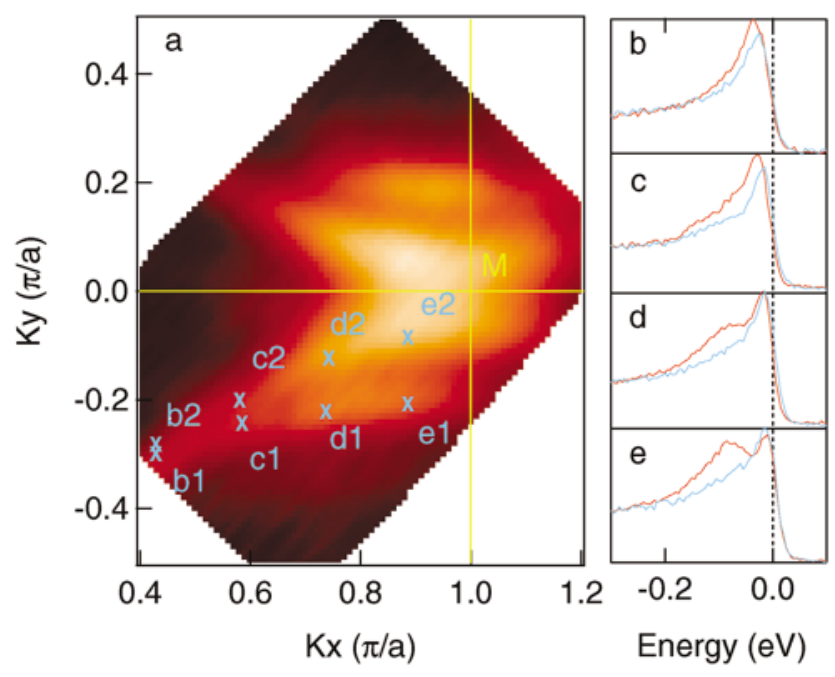

FIG. 3 (color). In this figure data collected at $100 \mathrm{~K}$ is shown. Panel (a) shows the spectral intensity at the Fermi level. Crosses indicate locations in momentum space of the cuts shown in panels (b)-(e). Blue spectra come from the bonding band, while red spectra come from the antibonding band. 
makes MDC peaks very broad and extracting width data is very complicated.

In Fig. 4 we plot the EDCs along the two Fermi surfaces with energy scales shifted to align all the peak positions, because we are only interested in peak widths. Panels (a) and (b) show normal state spectra. EDCs along the bonding band are shown in panel (a). The bottom spectra corresponds to the EDC at the Fermi surface closest to the $\Gamma-Y$ line $\left(\phi=45^{\circ}\right)$ while the topmost spectra corresponds to the EDC closest to the $\Gamma-M$ line $\left(\phi=0^{\circ}\right)$, as indicated by the black arrows in the inset. While the shape of the EDCs is complicated by the presence of the high energy hump due to the antibonding band, this does not effect the low energy part of the spectra, and we can use that part to estimate peak width change with angle by measuring the distance from the leading edge position to the peak position. The change in the peak width along the Fermi surface can be visually represented by the distance separating the two lines connecting the peaks and midpoints of the curves. One clearly sees the decrease in the peak width on going away from the node. In panel (b) we show the EDCs along the antibonding band. Again, we represent the change in peak widths along the Fermi surface by the distance between the two lines, and again we see a decrease in the peak width on going away from the node, similar to panel (a). In panels (c) and (d) we plot correspondingly bonding band and antibonding band EDCs from the Fermi surfaces in the superconducting state. The spectra cover the same parts of Fermi surfaces as EDCs in panels (a) and (b). Both bonding and antibonding bands show no increase towards $M$ point.

To further quantify the width data, we model the bonding band spectra near the Fermi surface by the sum of a Lorentzian peak to represent $A(\vec{k}, \omega)$ and a smooth function to represent inelastically scattered electrons (the results are insensitive to the form of the smooth function - we used parabola here, but linear and other functions work as well). This sum is then multiplied by the resolution-broadened Fermi function, where the chemical potential is a fitting parameter. A constant is added to the result to represent the signal from higher order photons. In fitting the antibonding band spectra, we simply add another Lorentzian to represent the bonding band hump. We present the widths from the fits of the bonding band spectra in the inset of panel (c) in Fig. 4. We see a clear trend, universal for both temperatures - the decrease in peak widths on approaching the $\phi=0$ line. Normal state data is plotted in open red squares and superconducting state data is plotted in filled blue squares. There are no data points close to $\phi=0$ line, because the bonding band does not come very close to the $\Gamma$ - $M$ line. Beyond the $\pm 20^{\circ}$ lines the bands are very close together, and the data points acquire large error bars, mostly coming from identifying the correct Fermi surface, and identification of the two overlapping features is

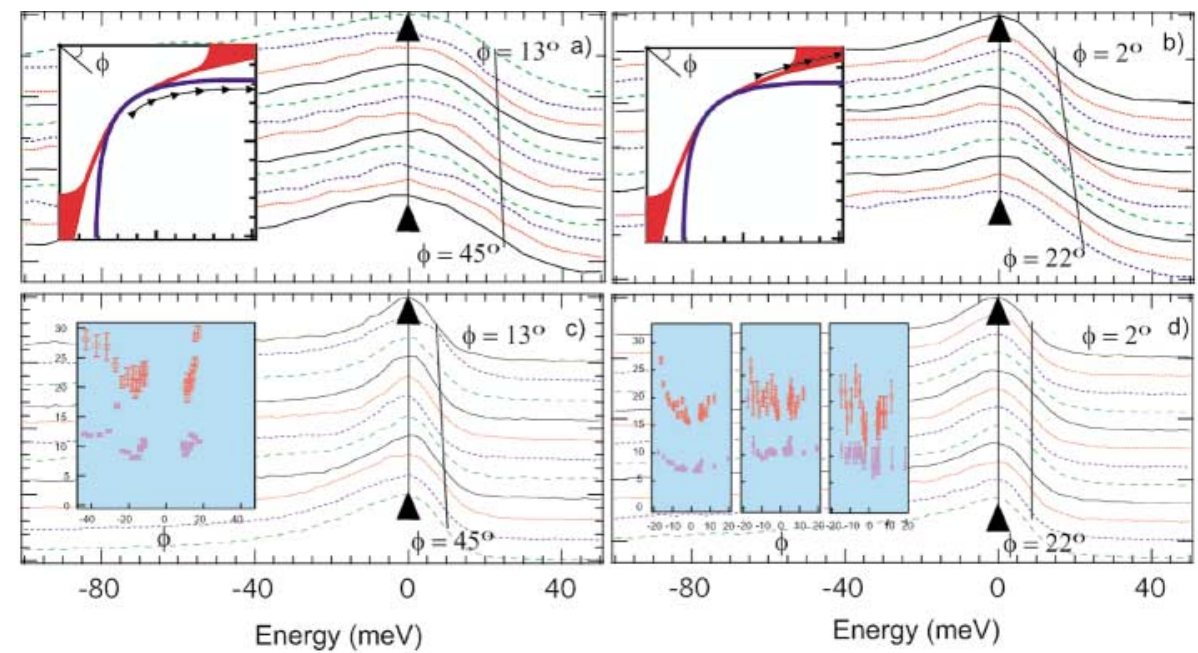

FIG. 4 (color). In this figure EDCs along the two Fermi surfaces are shown. Energy scale was shifted by constants to align all the peak positions. Panels (a) and (b) show normal state spectra. EDCs along the bonding band are shown in panel (a). The spectra are stacked bottom up in the direction from nodal direction towards the $(\pi, 0)$ point so that the scan near the antinode is on the top, as illustrated in the insets. EDCs along the antibonding band are shown in panel (b). Inset in panel (b) illustrates the stacking order of spectra. Panels (c) and (d) show superconducting state spectra on the Fermi surface. Stacking order is the same as in panels (a) and (b). Panel (c) shows bonding band spectra, while panel (d) shows antibonding band spectra. Inset in panel (c) shows full width half maximum (FWHM) of the bonding band peaks. Normal state data is plotted in open red squares and superconducting state data is plotted in filled blue squares. FWHMs are plotted as a function of $\phi$, the angle between the $\Gamma-Y$ direction and the line connecting a particular Fermi surface point to $\Gamma$, defined in the insets in panels (a) and (b). Inset in panel (d) shows FWHM of the antibonding band peaks. Three panels correspond to three different width extraction methods. As in the inset in (c), normal state data is plotted in open red squares and superconducting state data is plotted in filled blue squares as a function of $\phi$. 
thus unreliable. In the leftmost inset of panel (d), we plot the antibonding peak widths from the fits described above. We see a decrease of the widths near $M$ in the normal state. In the superconducting state there appears to be a shallow minimum at $M$, but the results are resolution limited. While the decrease in width towards $\pi, 0$ is less clear here, there is certainly no increase as expected from $Q=(\pi, \pi)$ scattering.

We have performed peak width measurements in two different Brillouin zone quadrants. The data in the insets of panels (c) and (d) in Fig. 4 comes from quadrant I, $K_{y}=-1$ to $K_{y}=0$ (negative $\phi$ ), and quadrant II, $K_{y}=0$ to $K_{y}=1$ (positive $\phi$ ). The same trend in the data from the two quadrants shows that the effects of remnant superstructure on the peak widths is negligible, because the superstructure effects are very different in the two quadrants.

To check whether our results are fitting model dependent, we plot in two more insets of panel (d) widths extracted in two more ways. In the middle panel, we plot the widths we obtained from fitting the antibonding data with the formula after [20] developed for the Bi2212 peakdip-hump spectra. In the rightmost panel, we plot the widths after measuring the distance from the leading edge midpoint to the peak. We see that all methods of data analysis give the same result - they show no width increase towards the $(\pi, 0)$ region. Taking into account nonuniform angular acceptance function of the electron analyzer does not change this result [21]. The lack of increase in the peak widths on approaching the $(\pi, 0)$ point demonstrates negligible effects of the $Q=(\pi, \pi)$ scattering, because the $Q=(\pi, \pi)$ scattering would lead to an increase in the widths near $(\pi, 0)$, at the intersection of the Fermi surface with the $(\pi, 0)-(0, \pi)$ line. This result contrasts sharply to the insulator [6,7] and underdoped [1-4] $p$-type case results and the NCCO $n$-type [5] result.

Anistropic inelastic spin scattering, referred to as a magnetic mode, was observed by neutron scattering measurements in several families of high temperature superconductors [22-25]. Some groups interpreted ARPES spectral features even in overdoped samples as resulting from the effect of the mode on the electronic structure $[14,16,17]$. This in turn invoked the possibility of coupling to magnetic mode as the pairing mechanism for superconducting electrons [14-16,26]. However, our results demonstrate that the effect of the $Q=(\pi, \pi)$ scattering in overdoped samples with high $T_{c}(70 \mathrm{~K})$ is negligible. This rules out scenarios that use the resonant magnetic mode as the pairing mechanism.

We thank J. D. Denlinger for the help with data analysis software. The experiment was performed at the Advanced Light Source of Lawrence Berkeley National Laboratory, supported by DOE Office of Basic Energy Science, Division of Materials Science with Contract No. DEAC03-76SF00098. The Stanford work was supported by an NSF grant through the Stanford MRSEC grant and
NSF Grant No. DMR-0071897. The SSRL's work was also supported by the Office's Division of Materials Science.

[1] Z.-X. Shen and J. R. Schrieffer, Phys. Rev. Lett. 78, 1771 (1997).

[2] A. Ino, Phys. Rev. B 62, 4137 (2000).

[3] J. C. Campuzano et al., Phys. Rev. Lett. 83, 3709 (1999).

[4] C. Kim et al., Phys. Rev. Lett. 80, 4245 (1998).

[5] N. P. Armitage et al., Phys. Rev. Lett. 87, 147003 (2001).

[6] B. Wells et al., Phys. Rev. Lett. 74, 964 (1995).

[7] F. Ronning et al., Science 282, 2067 (1998).

[8] D. L. Feng et al., Phys. Rev. Lett. 86, 5550 (2001).

[9] Y. D. Chuang et al., Phys. Rev. Lett. 87, 117002 (2001).

[10] P.V. Bogdanov et al., Phys. Rev. B 64, 180505 (2001).

[11] A. A. Kordyuk et al., cond-mat/0110379.

[12] A. Lanzara et al., Nature (London) 412, 510 (2001).

[13] H. Y. Kee et al., cond-mat/0110478.

[14] M. Eschrig et al., Phys. Rev. Lett. 85, 3261 (2000).

[15] Ar. Abanov et al., cond-mat/0112126.

[16] P. D. Johnson et al., Phys. Rev. Lett. 87, 177007 (2001).

[17] A. D. Gromko et al., cond-mat/0202329.

[18] O. K. Andersen et al., J. Phys. Chem. Solids 12, 1573 (1995).

[19] Here one does not see the double structure (sometimes called "peak-dip-hump" structure) expected from coupling with collective modes such as phonons [12]. Two factors contribute. First, the effect is weaker in overdoped samples [12]. Second, the effect is strong only when the quasiparticle has an energy comparable to that of a phonon, not at the Fermi energy as in this case [see, e.g., S. LaShell et al., Phys. Rev. B 61, 271 (2000)].

[20] A. Fedorov et al., Phys. Rev. Lett. 82, 2179 (1999).

[21] Angular resolution along the scan direction [parallel to $(0,0)-(\pi,-\pi)$ ] was almost a factor of 3 less than the resolution perpendicular to the scan direction. The effect of this rectangular angle acceptance relative to the Fermi surface can be important. We address this point by analyzing data from the two different quadrants. The scan direction always remained parallel to the $(0,0)-(1,-1)$ line. Let us consider two equivalent points in quadrants I and II (points mirror reflected with respect to the $K_{Y}=$ 0 line). Both points were detected with a large angular acceptance window along the $(0,0)-(1,1)$ line and a small acceptance window along the $(0,0)-(1,-1)$ line. However, the bands in quadrant II are mirror images of the bands from quadrant I with respect to the $K_{Y}=0$ mirror plane. It means that data from the second quadrant was taken with an angular acceptance function rotated $90^{\circ}$ with respect to the band structure. The fact that the results from the two quadrants are the same implies that the effect of angular acceptance in the data is small.

[22] J. Rossat-Mignod et al., Physica (Amsterdam) 235C, 59 (1994).

[23] H. F. Fong et al., Phys. Rev. Lett. 75, 316 (1995).

[24] P. Dai et al., Phys. Rev. Lett. 77, 5425 (1996).

[25] H. F. Fong et al., Nature (London) 398, 588 (1999).

[26] J. P. Carbotte et al., Nature (London) 401, 354 (1999). 